\title{
Propuesta pedagógica para el mejoramiento del clima organizacional
}

Diego Alejandro Cortés Rodríguez

Universidad Autónoma del Caribe

vulcanoodin@hotmail.com

https://orcid.org/0000-0002-6311-0675

MCs. Soledad Leal Pacheco

Universidad Autónoma del Caribe

sleal@uac.edu.co

https://orcid.org/0000-0002-9455-0788

\section{Cómo referenciar este artículo:}

Cortés-Rodríguez, Diego Alejandro; Leal Pacheco Soledad (2019) Conceptualización; Administración, Burocracia y Organización, ¿Es el management un sistema adaptativo complejo?, análisis desde las escuelas de pensamiento. Una aproximación preliminar a la temática. Revista Encuentros, Universidad Autónoma del Caribe. Vol. 17-01 de enero-junio.

DOI: http://dx.doi.org/10.15665/encuent.v17i01.1674

\section{RESUMEN:}

El clima organizacional a nivel empresarial ha sido ampliamente estudiado por carreras como la psicología social, organizacional y laboral y los estudios en administración. Estos estudios en su mayoría desarrollan metodologías para medir el clima dentro de determinada empresa o concluye la necesidad de mejorarlo en alguna de sus dimensiones por medio de estrategias de capacitaciones o talleres con los empleados. Sin embargo estos estudios rara vez hablan sobre la metodología pedagógica a implementar y mucho menos sobre la didáctica que se debe usar para fortalecer determinada dimensión del clima. Este artículo pretende explorar la aplicación de una estrategia pedagógica, particularmente basada en la pedagogía dialogante, aplicada mediante talleres secuenciales con herramientas didácticas tradicionales, sumadas y articulada a las encuestas, que contribuyeron a explorar los mecanismos aplicados para mejorar el trabajo en equipo al interior de una empresa de telecomunicaciones, basada en la forma en que aprenden los adultos y por medio del fortalecimiento de las competencias socio afectivas de los individuos

Palabras clave: Clima organizacional, didáctica de adultos, Trabajo Cooperativo, relaciones interpersonales

\section{Curriculum proposal for improving the organizational climate}

\begin{abstract}
Working environment in companies had been exhaustively studied by careers like psychology and Business administration. Mostly, this studies develop methodologies for measuring the environment in a particular company or recommends the implementation of training strategies or workshops with the employees. However, this studies rarely talk about the pedagogical methodology and the didactics that the companies should use for get stronger a particular dimension of the environment. This article pretends to explore the effectivity of an aditional pedagogical methodology to improve the team work within a telecommunications company based in the way that the adult people learn and the strengthening of their socio-emotional skills.
\end{abstract}

Keywords: organizational climate, adult teaching, cooperative work, interpersonal relations 


\title{
Proposta curricular para a melhoria do clima organizacional
}

\begin{abstract}
ABSTRATO
Ambiente de trabalhoem empresas tinham sido exaustivamenteestudada por carreiras como Psicologia e Administração. Principalmente, este estudos desenvolver metodologias para medir o ambiente emuma determinada empresa ourecomenda a implementação de estratégias de formaçãoouworkshopscom os empregados. No entanto, este estudos raramente falam sobre a metodologia pedagógica e didática que as empresas devem usar para ficarmaisforteumadimensão particular do meio ambiente. Este artigo pretende explorar a efetividade de umametodologia pedagógica para melhorar o trabalhoem equipe dentro de uma empresa de telecomunicaçõescom base namaneira que as pessoas adultas aprender e o reforço das suascompetênciassócio-emocionais.
\end{abstract}

Palavras-chave: clima organizacional, ensino de adultos, trabalho cooperativo, relaçõesinterpessoais.

\section{Introducción}

Una organización sólo existe cuando dos o más personas se juntan para cooperar entre sí y alcanzar objetivos comunes, que no pueden lograrse mediante iniciativa individual. (Chiavenato, 2007)

Toda empresa tiene como finalidad la consecución de objetivos tales como aumento del EBITDA, crecimiento, posicionamiento en el mercado, e incremento de ventas entre otras. Sin importar que tan automatizadas se encuentren las operaciones de la empresas, los objetivos propuestos siempre se alcanzan a través de personas que deben interactuar entre si y establecer relaciones laborales en medio de un entorno social demarcado por jerarquías y normativas. Adicionalmente el talento humano es considerado el activo más importante que posee una organización (Edvinsson, 1998) ya que son las personas las que agregan valor a cada uno de los productos y servicios que ésta genere y hacen productivos los recursos invertidos. De la satisfacción, bienestar o agrado con el ambiente en el que están inmersos los trabajadores y de la conexión de ese ambiente con sus metas personales y proyecto de vida, dependerá su desempeño aportando al cumplimiento de los objetivos estratégicos de la organización.

El clima laboral entendido como el estado de ánimo de la empresa se convierte entonces en un factor fundamental para alcanzar las metas y garantizar el sostenimiento y crecimiento de la misma. El comportamiento y la forma de relacionarse de un individuo al interior de una empresa, pueden afectar el desarrollo de la misma, su imagen en el mercado y su productividad. Desde esta perspectiva el clima laboral se convierte en otro activo de la empresa y como tal debe valorarse e invertir esfuerzos en maximizarlo.

El clima organizacional consiste en las percepciones compartidas de los atributos de una organización y sus subsistemas y en la influencia de dicha percepción sobre la relación de la organización con sus miembros y los grupos de interés. Así pues, el clima organizacional está relacionado con las características del líder, el cual ejerce un fuerte efecto sobre el mismo. El clima, a su vez, incide en la motivación, en el desempeño laboral y la salud de los trabajadores, así como en la eficiencia, satisfacción percibida, afecto al trabajo y con el nivel de ausentismo laboral (Uribe, 2010) 
Según García un buen clima laboral está caracterizado por el entusiasmo, el optimismo y el trabajo en equipo, y se traduce en unas etapas que vive cada integrante, comenzando por el conocimiento interior de sí mismo, siguiendo por la expresión y la exteriorización de estas ideas y terminando con el trabajo creativo desarrollado y su posterior implementación. Es por esto, que una de las ventajas de propiciar un buen clima al interior de la organización es que permite desarrollar en el sujeto, de forma más libre y acertada, todo el potencial que éste tenga y aprovecharlo en beneficio de la empresa, (Garcia, 2004)

Se hace necesario mejorar el clima organizacional por medio del fortalecimiento de las relaciones sociales-laborales que aumenten la felicidad del trabajador dentro de la empresa y su percepción favorable del entorno. Por tal motivo, Arguile Michael (1987) expresa:

Las relaciones sociales son una fuente fundamental de felicidad, de disminución de la angustia y de salud. Los mayores beneficios provienen del matrimonio y de otras relaciones íntimas de confianza y de apoyo [...] Las relaciones aumentan la felicidad porque producen alegría, proporcionan ayuda y realizan actividades agradables en común. Redu- cen los efectos de la tensión mediante el incremento de la autoestima, la eliminación de las emociones negativas y la provisión de ayuda para resolver "problemas (p. 50).

En Claro Colombia con sede en Medellín, los trabajadores cuentan con los espacios físicos y de tiempo para que reciban toda la capacitación necesaria para desempeñar las funciones propias de su cargo de manera óptima tanto al ingresar a la empresa, durante su entrenamiento al cargo, cómo al momento de requerir una instrucción o actualización ya sea técnica o procedimental. Sin embargo, y desde una visión holística e integral Amezola (2008), plantea que la formación promovida por la institución educativa (en este caso la empresa apoyada en formadores internos y externos) no sólo debe diseñarse en función de la incorporación del sujeto a la vida productiva a través del empleo, sino más bien, como dice Andrew Gonczi (1996):

partir de una formación profesional que además de promover el desarrollo de ciertos atributos (habilidades, conocimientos, actitudes, aptitudes y valores), considere la ocurrencia de varias tareas (acciones intencionales) que suceden simultáneamente dentro del contexto (y la cultura del lugar de trabajo) en el cual tiene lugar la acción; y a la vez permita que algunos de estos actos intencionales sean generalizables (p. 265-288).

Pedagogos como Miguel de Zubiría Samper (2009), plantean que hoy es posible apuntar hacia la felicidad si hacemos especial énfasis en el estudio de las competencias sociales fuente fundamental de felicidad, de disminución de la angustia y privilegio de la salud.

Humberto Maturana (2002) expresa que:

los seres humanos, en sentido estricto, surgimos del amor, porque el amor como emoción constituye el dominio de acciones de aceptación recíproca en el que pudo surgir y conservarse el lenguaje, añadiéndose al modo de vida de nuestros ancestros homínidos como parte constitutiva del vivir que nos define (p. 45).

El presente trabajo nace del análisis ordenado de una herramienta de diagnóstico que para el caso es la encuesta propuesta por el autor Carlos Eduardo Mendez en su instrumento IMCOC (Instrumento de medición del Clima Organizacional en Colombia). Después de la aplicación de la encuesta surgen diversos interrogantes a los cuales se quiere dar respuesta dentro del desarrollo 
de la investigación. Interrogantes como:

¿Qué dimensiones de las evaluadas en la encuesta IMCOC pueden ser intervenidas de manera positiva mediante la implementación de una estrategia de enseñanza - aprendizaje? ¿Qué aspectos intrapersonales e interpersonales influyen negativamente en los trabajadores de la empresa?

¿Cómo articular la estrategia seleccionada dentro de los procesos de capacitación normales de la empresa?

Interrogantes a los cuales se les pretende dar respuesta durante el desarrollo de la investigación y a partir de los cuales surgen los siguientes objetivos para el proyecto:

Objetivo general: Aplicar una estrategia de enseñanza aprendizaje para complementar desde la didáctica, el mejoramiento del clima organizacional de los empleados de la empresa Claro Colombia de la ciudad de Medellín.

Objetivos específicos: Identificar que dimensiones dentro de la encuesta de clima laboral IMCOC pueden intervenirse positivamente.

Identificar los aspectos intrapersonales e interpersonales que influyen negativamente en los trabajadores de la empresa.

Aplicar como prueba piloto la estrategia escogida dentro de los planes cotidianos de formación.

\section{DESARROLLO TEMÁTICO Marco referencial teórico Cultura Organizacional}

Siguiendo a Rousseau (1990), los investigadores organizacionales coinciden en definir la cultura como conjuntos de cogniciones compartidas por los miembros de una determinada unidad social, los cuales se adquieren a través del aprendizaje social y de procesos de socialización que exponen a los individuos a diversos elementos culturales, como actividades e interacciones, informaciones comunicadas y artefactos materiales, que conforman la experiencia social al tiempo que dotan a sus miembros de valores compartidos, marcos de comprensión comunes y sistemas de creencias y de expectativas. No obstante, las culturas no son uniformes ni estáticas (Goffee \& Jones, 2001), ya que cambian y se configuran de manera dinámica a lo largo del tiempo. (Jaime \& Cabrera, 2007, p.301).

Por su parte, Trice y Beyer (1993) consideran que la cultura humana emerge a partir de los esfuerzos desplegados por sus miembros para afrontar incertidumbres y ambigüedades de todo tipo, proporcionándoles individual y colectivamente conjuntos más o menos articulados de ideas que les permitan responder a sus experiencias. Es decir, que todo constructo cultural se identifica a través de creencias compartidas, que se interrelacionan a causa de las carga emocional y comunicacional que surge de las experiencias, o de las afecciones que llegan a la conciencia humana. Este estado de afección es posible gracias a la comunicación como método.

En el campo empresarial es fundamental motivar a las personas en sus puestos de trabajo, es esencial si se quiere ser competitivo, ya que el desarrollo de la empresa y el logro de los objetivos depende directamente del capital humano y del trabajo en equipo. La motivación se compone básicamente por las necesidades, impulsos e incentivos, es decir las necesidades nacen cuando hay una carencia ya sea psicológica o física y varían de acuerdo a la cultura o la forma de ser de las persona; ahora de acuerdo a expertos los impulsos son los que alimentan la necesidad creando en la persona la toma de decisión entre elegir y realizar una acción, los incentivos satisfacen una necesidad; Rodríguez (1988) lo considera como "El conjunto de razones que explican los actos de un individuo", es decir, y complementándolo con la perspectiva de Norma Pérez, consideramos dentro de este todo los impulsos, necesidades e intereses que llevan a las persona a actuar de cierta manera. (Pérez, 
Figura 1. Adaptado de Chapman (2007).

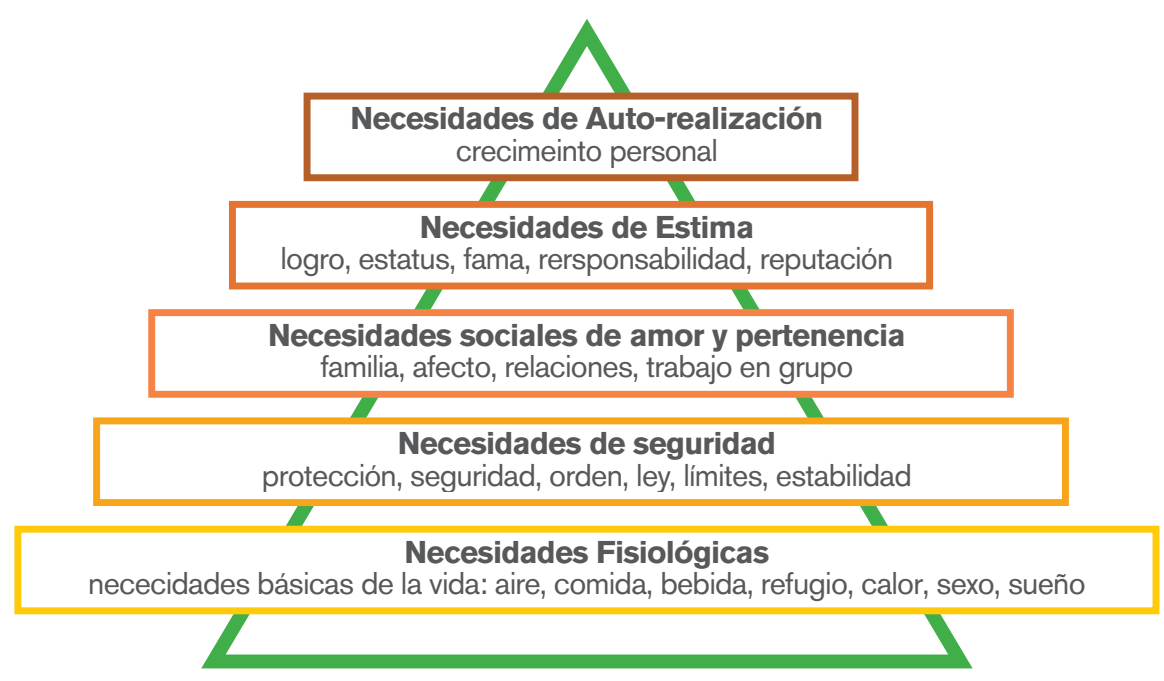

\section{4, p. 51).}

La "Teoría de la Motivación Humana”, propone una jerarquía de necesidades y factores que motivan a las personas; esta jerarquía se modela identificando cinco categorías de necesidades y se construye considerando un orden jerárquico ascendente de acuerdo a su importancia para la supervivencia y la capacidad de motivación. De acuerdo a este modelo, a medida que el hombre satisface sus necesidades surgen otras que cambian o modifican el comportamiento del mismo; considerando que solo cuando una necesidad está "razonablemente" satisfecha, se disparará una nueva necesidad (Colvin y Rutland 2008).

La figura 1, muestra la jerarquía de necesidades propuesta por Maslow representadas en forma de una pirámide.

Según Maslow (1991), las necesidades orientan la conducta ya que el individuo busca la forma de satisfacerlas. Él distingue 5 necesidades y considera las fisiológicas y de seguridad como de orden inferior y se satisfacen en el exterior ejemplo (sueldos, antigüedad), las tres siguientes se clasifican como de orden superior que se satisfacen en el interior del individuo.

\section{El ser humano ser caracteriza} por esencialmente establecerse y formarse como ser social, es acá cuando hablamos de que la naturaleza del hombre implica constituir relaciones de cooperación y afiliación enfocadas en el logro. En el contexto de las organizaciones se ha venido destacando a través de los años la importancia de un buen
Clima Organizacional y de una óptima gestión del talento humano; cuando hablamos de este tema debemos analizar que existen una seria de factores que influyen en el clima laboral de los empleados entre los que podemos encontrar los diferentes tipos de liderazgo, la forma en cómo se implementan y definen los objetivos, la gestión y procesos relacionados con la toma de decisiones. Internamente también hacemos referencia a los proceso de comunicación que determinan parámetros y conductas que son propias de los miembros de la organización y que Darío Mansilla (2007) expresa:

que las conductas y los patrones de comportamiento de los individuos en la organización (sistema de personalidad) son el resultado del conjunto de valores, normas y pautas propias de la estructura organizacional(sistema cultural), como de las condiciones que se establecen por el proceso de interacción, sistema social (p. 43).

En el entorno laboral la motivación se debe dirigir a conseguir que los empleados alcancen un grado de excelencia en el trabajo, es preciso que se deba hacer énfasis en que cada empleado disfrute de la realización de su trabajo, diseñe formas para hacerlo cada vez mejor y de manera práctica de 
este modo se asegura la calidad y eficiencia en el cumplimiento de tareas. Diferentes teorías y modelos han estudiado la motivación y como esta influye en el comportamiento del individuo, la mayoría de estas se concentran en las necesidades. (Álvarez, 2003)

\section{Clima Organizacional}

Se define el clima organizacional como una mezcla delicada de interpretaciones o percepciones, que en una organización hacen las personas de sus trabajos o roles. Son las percepciones de los miembros del grupo las que definen el clima, y solo a partir de esas percepciones se podrá conocer y determinar las características de ese clima organizacional. (Cornell, 1955)

Argyris (1957), hace énfasis en el desarrollo de una atmósfera interpersonal de confianza, franqueza y tranquilidad en la organización para que se pueda aceptar la existencia de un conflicto cuando este se presenta, identificarlo y emplear los recursos necesarios para resolverlo.

Atkinson (1964), creó un modelo con el cual explicaba la "motivación promovida", un efecto de los motivos íntimos del individuo, de los incentivos que la organización le provee y de las experiencias despertadas en la relación, como un elemento moldeador del clima organizacional.
El ambiente interno en que se encuentra la organización lo forman las personas que lo integran, a esto denominamos Clima organizacional, que involucra los sentimientos psicológicos que manifiestan el funcionamiento interno de la organización, de tal manera que la forma de comportarse de un individuo en el trabajo no depende de su forma de ser y características personales, sino también de cómo se percibe su Clima de trabajo y el entorno. (Duque Delgado, 2009)

Citando estudios sobre clima organizacional realizados en Colombia Díaz Pinilla (2010) encuentra que "a cada uno de los niveles, las relaciones entre la dirección y el personal son mejores, cuando la comunicación no se hace solamente de manera ascendente o descendente, sino también de forma lateral, los empleados están motivados para la participación y la implicación, por el establecimiento de objetivos de rendimiento". En este sentido de verticalidad u horizontalidad, quien es jefe determina el clima de manera jerarquizada pero siempre objetivando sus posibilidades de relación con aperturas, cerramientos, fraternizaciones, familiarizaciones y autonomías.

Díaz Pinilla (2010) propone 4 tipos de clima organizacional.

Clima tipo autoritario explotador: La dirección no tiene confianza en sus empleados, la mayor parte de las decisiones y de los objetivos se toman en la cima de la organización. Los empleados tienen que trabajar dentro de una atmosfera de miedo, de castigos, de amenazas, ocasionalmente de recompensas, y la satisfacción de las necesidades permanece en los niveles psicológicos y de seguridad.

Clima tipo autoritario paternalista: Es aquel en que la dirección tiene confianza condescendiente en sus empleados. La mayor parte de las decisiones se toman en la cima, pero algunas se toman en los escalones inferiores; sin embargo genera impresión de trabajar dentro de un ambiente estable y estructurado.

Clima tipo participativo consultivo: Las decisiones se toman generalmente en la cima pero se permite a los subordinados que tomen decisiones más específicas en los niveles inferiores, la comunicación es de tipo descendente, las recompensas, los castigos ocasionales, se trata de satisfacer las necesidades de prestigio y de estima.

Clima tipo participativo en grupo: Los procesos de toma decisiones están diseminados en toda la organización y muy bien integrados a cada uno de los niveles, las relaciones entre la dirección y el personal son mejores, los empleados están motivados para la participación y la implicación, por el establecimiento 
de objetivos de rendimiento, se trata de satisfacer las necesidades de auto realización y autoestima.

\section{Enseñanza aprendizaje}

En el contexto educacional el proceso de comunicación es muy importante ya que se convierte en el elemento que intermedia entre el docente y el alumno. En palabras de Claudia Castañeda (2014) citando a Goleman (1997):

La comunicación es hacer común a dos o más individuos, un contenido semántico cualquiera por medio de diversos recursos técnicos: lenguaje oral, imágenes gráficas y visuales, entre otras (...) La esencia humana no es algo abstracto, inherente al individuo, sino que es el conjunto de todas las relaciones sociales (p. 23).

Para Mario Kaplún (1997) al comunicar se aprende, los emisores y receptores aprenden unos de otros, tanto en los procesos educativos como en los comunicativos, pues el lenguaje es el instrumento del pensamiento, a partir del cual no solamente podemos comunicarnos, sino también pensar. ¿Cómo se adquiere el lenguaje? ¿Cómo aprendemos la cultura?: Comunicándonos.

\section{IMCOC}

Creado en 1980 por Carlos Méndez, sus siglas significan: Ins- trumento para Medir el Clima en Organizaciones Colombianas, y fue presentado en su libro Clima Organizacional en Colombia (2006). El IMCOC es un método de análisis que es útil para obtener resultados idóneos en los estudios de cultura organizacional realizados a 176 empresas colombianas entre 1980 y 2005.

El IMCOC es una herramienta que ayuda a directivos a tomar decisiones para mejorar la cultura organizacional de sus empresas.

Entre sus características están la de ser acumulativo en los resultados encontrados, sus teorías son generalizaciones empíricas, explica y predice, y es aplicable. Este instrumento está conformado por 45 preguntas y un software para el procesamiento de la información, y así encuentra las variables primordiales para mejorar el clima organizacional que son: cooperación, relaciones interpersonales, toma de decisiones, liderazgo, motivación, objetivos y control, según las 13.000 encuestas realizadas (Méndez 2006).

La encuesta presenta en 7 categorías los conceptos fundamentales sobre los cuales propone evaluar el clima organizacional:

Categoría No 1: objetivos. Esta categoría hace referencia al conocimiento que tienen los empleados sobre la misión, visión, objetivos y funcionamiento de la empresa. No es resultado del proceso de inducción, sino que se adquiere por la antigüedad y la experiencia, advierte el investigador Méndez.

\section{Categoría No 2: cooperación.}

Definida como el sentimiento de los miembros de la empresa sobre la existencia de un espíritu de ayuda de parte de los directivos y de otros empleados del grupo. Su énfasis está puesto en el apoyo mutuo, tanto de niveles superiores como inferiores.

\section{Categoría No 3: relaciones} interpersonales. Esta categoría es definida por los autores como la percepción por parte de los miembros de la empresa acerca de la existencia de un ambiente de trabajo grato y de buenas relaciones sociales entre todos los empleados de la organización. Las relaciones de carácter personal, sustentadas en actividades de tipo informal (recreación, deportes y otros), no tienen relevancia para los empleados en sus procesos de relaciones sociales. Sin embargo, las califican de ,alguna importancia $\mathbb{\bigotimes}$ cuando estas actividades son organizadas o dirigidas desde la empresa o por los compañeros de área de trabajo.

Categoría No 4: liderazgo. Esta categoría es definida por el autor como la capacidad de las directivas y de los seguidores para asumir desafíos y grandes cambios hacia 
objetivos comunes, definición desde la cual surgen diferentes estilos de liderazgo.

Categoría No 5: control. Definida como aquella que representa la percepción que tienen los miembros de la organización acerca de la cantidad de reglas, procedimientos, trámites y otras limitaciones a los que se ven enfrentados en el seguimiento de su trabajo o de sus tareas.

Categoría No 6: toma de decisiones. Corresponde al sentimiento que tienen los miembros de la organización acerca de los desafíos que impone el trabajo y la participación de las personas en las decisiones de la empresa. Es la medida en que la organización promueve la aceptación de riesgos calculados a fin de lograr los objetivos propuestos, y una correlación entre el estilo de liderazgo y la forma como las personas participan en las decisiones de la empresa.

Categoría No 7: motivación. Está determinada por los diferentes comportamientos que evidencian los individuos tras su persecución de logros y metas con el fin de llegar a satisfacer sus diferentes necesidades.

\section{Escala de Likert}

Consiste en un conjunto e ítems presentados en forma de afirmaciones o juicios ante los cuales se pide la reacción de los sujetos. Es decir, se presenta cada afirmación y al sujeto que externe su reacción eligiendo uno de los cinco puntos de la escala. A cada punto se le asigna un valor numérico. Así, el sujeto obtiene una puntuación respecto a la afirmación y al final se obtiene su puntuación total sumando las puntuaciones obtenidas en relación a todas las afirmaciones. Las afirmaciones califican al objeto de actitud que se está midiendo y deben expresar sólo una relación lógica, además es muy recomendable que no excedan de 20 palabras. (Fernández 2006).

\section{Metodología}

El diseño metodológico de la investigación es cuantitativo en el cual se trabajan dos grupos intactos uno de ellos de control con la aplicación de un pretest y postest y el otro experimental donde se aplicaron ambos test y se desarrolló la metodología propuesta.

Cómo métodos de investigación teóricos se utilizaron el análisis y la síntesis para el procesamiento de la información y sacar conclusiones.

Los métodos y técnicas empíricas utilizadas son: observación, encuestas, y revisión documental para el diagnóstico del problema.
El instrumento se diseñó con base a el IMCOC de Carlos

Eduardo Mendez Álvarez donde se tomaron en cuenta solo las dimensiones de Cooperación y Relaciones Interpersonales, por cuanto pueden ser las intervenidas con estrategias de tipo pedagógico ya que constituyen parte del aprendizaje organizacional. Las dimensiones Objetivos, Liderazgo, Toma de decisiones, Motivación y Control no fueron tenidas en cuenta para esta investigación ya que dependen de múltiples factores que no pueden ser impactados desde la estrategia pedagógica, como salarios, estado de herramientas de trabajo, reconocimiento, visión de la organización y otras inherentes al puesto de trabajo.

El cuestionario se aplicó a 80 trabajadores directos de la empresa Claro Colombia con sede en la ciudad de Medellín, 40 del grupo de control los cuales fueron escogidos al azar y 40 del grupo experimental a los cuales se les invitó y accedieron voluntariamente a participar de los talleres. Los trabajadores pertenecen a las áreas de proyectos, comercial, servicio, mantenimiento, operaciones, logística y corporativo cómo muestra la tabla 1 . 
Tabla 2. Elaboración propia.

Los databla de Elaboración propiąnto Ákarasel grupo dGrupQtrol cóGrupo para el grupe de Control Expe expentmental camercia on la hejrramienta ${ }_{3}^{3} x c e l$ servicio

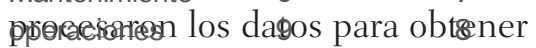
Cosrperativeados. $3 \quad 1$ Logística 2 ación 1 Goyeformación de los tallenes desarroltados en la estrategia de enseñanza aprendizaje.

Con el fin de apalancar los procesos de aprendizaje y adaptación al entorno organizacional, se exploró las competencias de Cooperación y Relaciones Interpersonales con una serie de actividades pedagógicas basadas en la pedagogía dialogante y técnicas de interacción grupal, donde la cooperación, la comunicación y el respeto incluyente se convierten en los pilares de la interacción.

Los referentes teóricos que se usaron como base para el diseño de los talleres fueron la inteligencia emocional de Daniel Goleman, la filosofía tolteca adaptada por Miguel Ruiz, las inteligencias múltiples de Howard Gardner, la ontología del lenguaje explicada por Rafael Echavarría, la pedagogía dialogante de Julián de Zubiría la didáctica para aprendizaje de adul-

\begin{tabular}{|c|c|c|c|}
\hline Taller & Nombre & Objetivo & Actividades \\
\hline 1 & Conocernos & Identificar los valores del equipo & $\begin{array}{l}\text {-Dinámica de presentación } \\
\text {-Lectura acuerdo } 1 \\
\text {-Mesa Redonda de socialización }\end{array}$ \\
\hline 2 & Inclusión & Aprender a tolerar las diferencias & $\begin{array}{l}\text {-Dinámica Rally } \\
\text {-Debate } \\
\text { "Lectura Acuerdo } 2\end{array}$ \\
\hline 3 & Interconexión & $\begin{array}{l}\text { Aprovechar la pluralidad } \\
\text { en beneficio del grupo }\end{array}$ & $\begin{array}{l}\text {-Inteligencia colectiva } \\
\text {-Lectura Acuerdo } 3 \\
\text {-Video la tierra del olvido }\end{array}$ \\
\hline 4 & Caos y Conflicto & Aprender a mediar conflictos & $\begin{array}{l}\text {-Ejercicio empresa ABC } \\
\text {-Juego, gallina ciega } \\
\text {-Lectura Acuerdo } 4\end{array}$ \\
\hline \multicolumn{2}{|c|}{5 Hagamos Acuerdos } & $\begin{array}{c}\text { Buscar el beneficio } \\
\text { de todas las partes } \\
\text { mediante la negociación }\end{array}$ & $\begin{array}{l}\text {-Juego resérvame una cita } \\
\text {-Lectura Ontología del lenguaje pag. } 189 \\
\text {-Actividad Visualizando el futuro }\end{array}$ \\
\hline \multicolumn{2}{|c|}{6 Estrategia de Equipo } & $\begin{array}{l}\text { Caracterizar al equipo } \\
\text { mediante una misión, } \\
\text { visión y estrategia }\end{array}$ & $\begin{array}{l}\text {-Conferencia magistral } \\
\text {-Lluvia de ideas } \\
\text {-Taller de estrategia }\end{array}$ \\
\hline 7 & Proyecto & $\begin{array}{l}\text { Poner en práctica } \\
\text { lo aprendido en los seis } \\
\text { talleres anteriores }\end{array}$ & $\begin{array}{l}\text {-Video Trabajo en equipo } \\
\text { "Lluvia de Ideas } \\
\text {-Desarrollo del proyecto }\end{array}$ \\
\hline
\end{tabular}

tos de George Homans y el aprendizaje experiencial de David Kolb.

La metodología de estudio aplicada está compuesta de cinco actividades: dinámicas en grupo, debates, lecturas, charlas magistrales y juegos. Estas se realizaron en grupos de 12 personas los cuales recibieron cada taller de manera semanal con una duración de 90 minutos, hasta completar los 7 talleres.

El contenido de los talleres se presenta de manera resumida en la tabla 2.

\section{Encuesta}

Las preguntas que se realizaron fueron en total 16 de las cuales las primeras 10 corresponden a la dimensión de cooperación y las 6 restantes a Relaciones Interpersonales.

La encuesta se encuentra en la tabla 3.

\section{Análisis de resultados}

Para el análisis de los datos se utilizó el método de escalonamiento de Likert con las opciones de respuesta adaptadas al contexto de la pregunta, por esta razón las opciones de respuesta eran: nunca, casi nunca, 


\begin{tabular}{|c|c|c|c|c|c|}
\hline $\begin{array}{l}\text { 1. ¿Califique la ayuda y la colaboración que usted cree que } \\
\text { existe entre los trabajadores de esta empresa? }\end{array}$ & Nunca & Casi nunca & Algunas veces & Casi siempre & Siempre \\
\hline $\begin{array}{l}\text { 2. ¿En qué grado usted, ayuda y colabora con sus compañeros } \\
\text { en el trabajo? }\end{array}$ & Nunca & Casi nunca & Algunas veces & Casi siempre & Siempre \\
\hline $\begin{array}{l}\text { 3. ¿Cómo califica su participación, si usted está vinculado en } \\
\text { grupos de trabajo en la empresa? }\end{array}$ & Muy mala & Mala & Regular & Buena & Muy buena \\
\hline $\begin{array}{l}\text { 4. ¿Califique la frecuencia con que usted acostumbra divertirse } \\
\text { con compañeros de su sección o de otra sección de la empresa? }\end{array}$ & Nunca & Casi nunca & Algunas veces & Casi siempre & Siempre \\
\hline $\begin{array}{l}\text { 5. ¿La empresa organiza paseos, actividades deportivas, fiestas } \\
\text { u otras actividades de diversión? }\end{array}$ & Nunca & Casi nunca & Algunas veces & Casi siempre & Siempre \\
\hline $\begin{array}{l}\text { 6. ¿Cómo califica usted, su participación en las actividades de } \\
\text { diversión que realiza su empresa? }\end{array}$ & Muy mala & Mala & Regular & Buena & Muy buena \\
\hline $\begin{array}{l}\text { 7. ¿Qué tanta ayuda le piden sus compañeros para desempeño } \\
\text { de su trabajo? }\end{array}$ & Nunca & Casi nunca & Algunas veces & Casi siempre & Siempre \\
\hline $\begin{array}{l}\text { 8. ¿Qué tanto participa usted, de las actividades que realizan } \\
\text { sus amigos en la empresa? }\end{array}$ & Nunca & Casi nunca & Algunas veces & Casi siempre & Siempre \\
\hline $\begin{array}{l}\text { 9. ¿Fuera de las horas de trabajo, con qué frecuencia se } \\
\text { relaciona usted con sus compañeros de labor? }\end{array}$ & Nunca & Casi nunca & Algunas veces & Casi siempre & Siempre \\
\hline $\begin{array}{l}\text { 10. ¿Califique la ayuda que usted, presta para la solución de los } \\
\text { problemas en su sección? }\end{array}$ & Muy mala & Mala & Regular & Buena & Muy buena \\
\hline $\begin{array}{l}\text { 11. ¿Cómo califica sus relaciones con sus compañeros de } \\
\text { trabajo? }\end{array}$ & Muy mala & Mala & Regular & Buena & Muy buena \\
\hline $\begin{array}{l}\text { 12. ¿Cuándo usted tiene un problema de trabajo lo soluciona } \\
\text { con sus compañeros y superiores? }\end{array}$ & Nunca & Casi nunca & Algunas veces & Casi siempre & Siempre \\
\hline 13. ¿Cómo califica usted, el trato y relación con su jefe? & Muy mala & Mala & Regular & Buena & Muy buena \\
\hline $\begin{array}{l}\text { 14. ¿Cómo califica usted, la confianza entre los jefes y } \\
\text { trabajadores de esta empresa? }\end{array}$ & Muy mala & Mala & Regular & Buena & Muy buena \\
\hline $\begin{array}{l}\text { 15. ¿Cuándo hay cambios en la empresa, en qué medida recibe } \\
\text { información al respecto? }\end{array}$ & Nunca & Casi nunca & Algunas veces & Casi siempre & Siempre \\
\hline $\begin{array}{l}\text { 16. ¿En qué medida se enteran las directivas de los problemas } \\
\text { en su sección? }\end{array}$ & Nunca & Casi nunca & Algunas veces & Casi siempre & Siempre \\
\hline
\end{tabular}

Tabla 2. Elaboración propia.

algunas veces, casi siempre y siempre o muy mala, mala, regular, buena y muy buena según el caso. A cada pregunta se le dio un valor siendo (1) el menos favorable y (5) el más favorable a la calificación. Cada puntaje obtenido se clasificó dentro un criterio a saber: 40= "Muy crítico", $80=$ "Crítico", 120= "Neutro", 160=
"Favorable", 200= "Muy favorable". Cada uno de los puntajes obtenidos se ubicó entre 2 de los criterios mencionados.

\section{Grupo intacto de control}

Al realizar el análisis del grupo intacto de control se observó que no hay mejora significativa entre el pretest y el postest y que el 50\% de los ítems de la encuesta mostró una disminución en su medición. Esto pudo haberse dado gracias a cambios internos que se han generado en el último año al interior de la organización.Este resultado afirma la necesidad de intervenir a los trabajadores con formación que mejore 
el relacionamiento y el trabajo en equipo. Citando a el padre del management Peter Drucker (1995) "Solo tres cosas ocurren de manera natural en las organizaciones: la fricción, la confusión y el bajo rendimiento. En todo lo demás hace falta Liderazgo". No se puede entonces esperar a que el clima laboral mejore por sí mismo, ya que la tendencia es a desmejorar y a que los conflictos y la falta de comunicación se agudicen.

En general los puntajes analizados en el pre y post test muestran una ligera disminución del $2 \%$ en la calidad del trabajo cooperativo y relacionamiento de las personas encuestadas y como lo muestra la figura 2 no hay un test que se haya comportado superior al otro.

Figura 2.

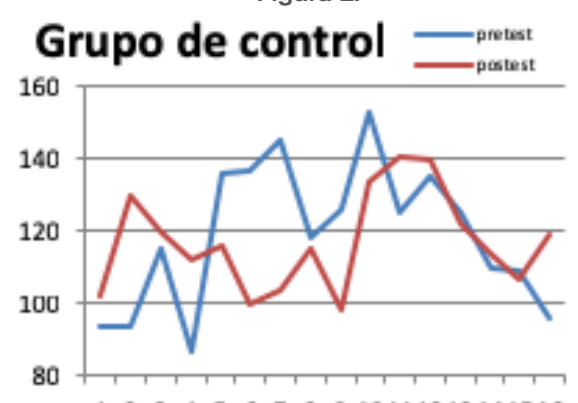

trabajo?

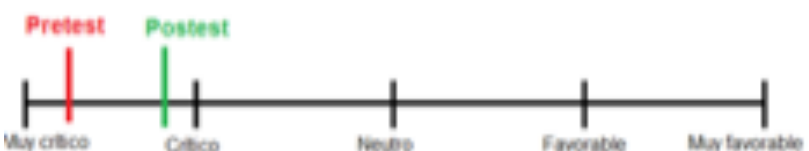

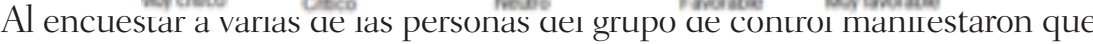
la colaboración entre compañeros de diferente área no es algo común sin embargo lo ${ }^{-1}$ pretést $\ldots$

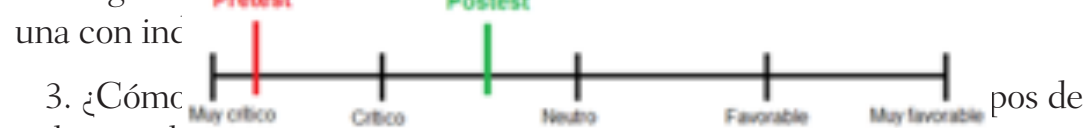
trabajo en la empresa?

4. ¿Califique la frecuencia con que usted acostumbra divertirse con compañeros de su sección o de otra sección de la empresa?

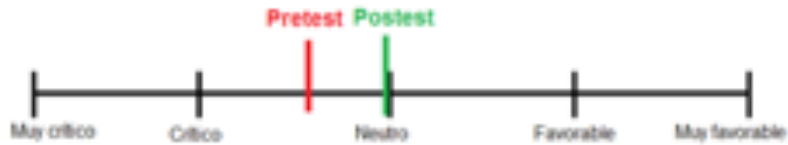

5. ¿La empresa organiza, paseos, actividades deportivas, fiestas u otras actividades de diversión?

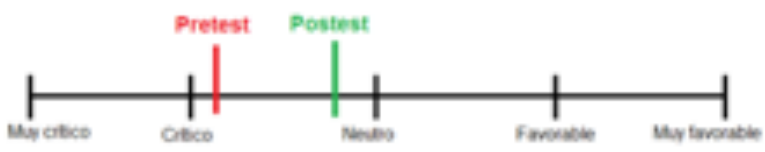

6. ¿Cómo califica usted, su participación en las actividades de diversión que realiza su empresa?

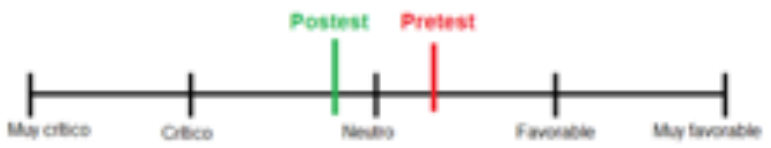

Varias de las personas encuestadas manifiestan no asistir a actividades programadas por la empresa o por compañeros de trabajo ya que no sienten una

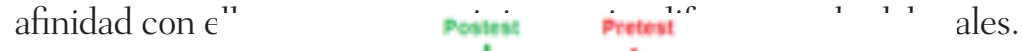

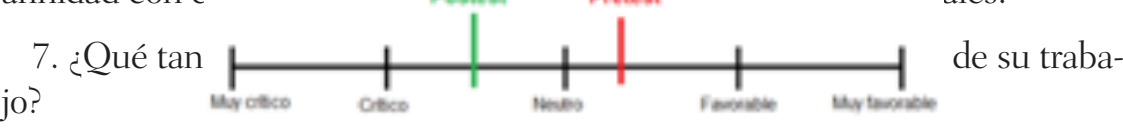


Algunos de los encuestados mar de trabajo razón por la cual asume por ende no piden ayuda.

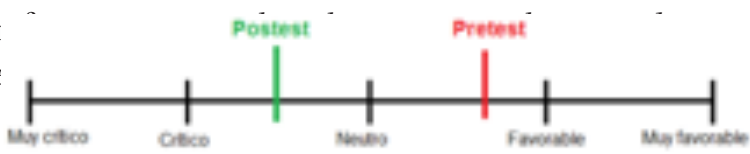

entos significativos en el volumen mo para ayudarles en sus tareas y

8. ¿Qué tanto participa usted, de las actividades que realizan sus amigos en la empresa?

9. ¿Fuera de las horas de trabaj

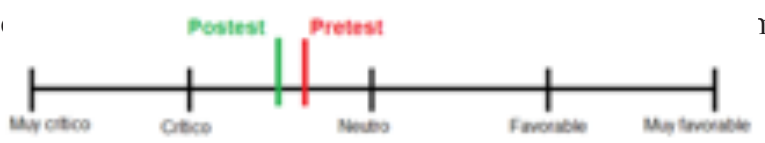

npañeros de labor?

10. ¿Califique la ayuda que ustı

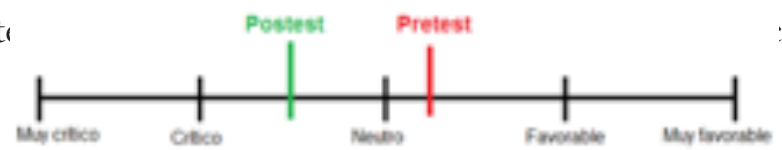

:ción?

En general los encuestados ma requiera, en caso de ser personas se encuentren.

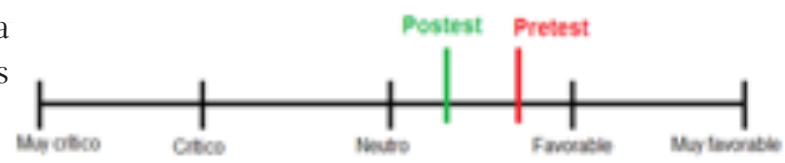

:ompañero de la misma área lo requieran y en qué tan ocupados

11. ¿Cómo califica sus relaciones con sus compañeros de trabajo?

12. ¿Cuándo usted tiene un pr

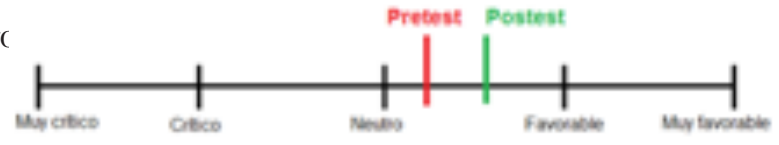

superiores?

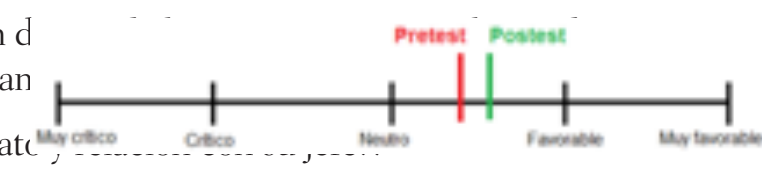

ca relación e incluso no conocer a

El trabajo en equipo es común d compañeros de otras áreas que tan

13. ¿Cómo califica usted, el trate

Ottes $\ldots . .$. 
14. ¿Cómo califica usted, la confianza entre los jefes y trabajadores de esta empresa?

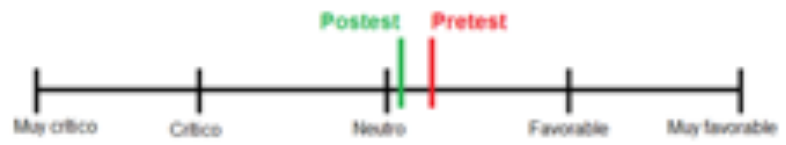

La relación con mandos superiores es buena, el buen trato y el respeto son constantes, $r$ que sus jefes d

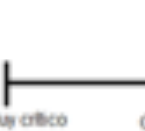
Pretest postes:
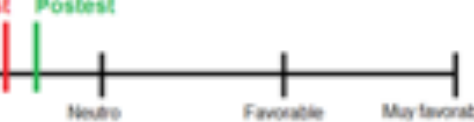
os siente pierda la confianza y se perciba una falta de comunicación vertical.

15. ¿Cuándo hay cambios en la empresa, en qué medida recibe información al respecto?

16. ¿En qué medida se enteran las directivas de los problemas en su sección?
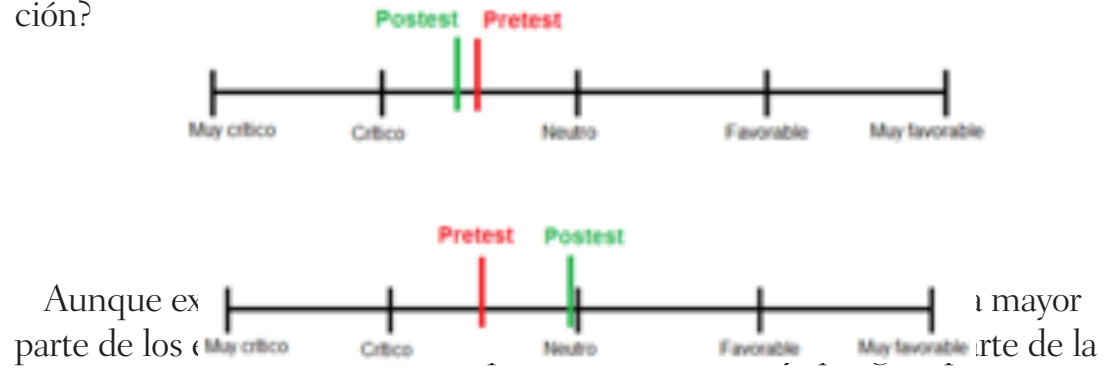

información que es relevante para su puesto no se comunica adecuadamente. Gracias a esta falta de confianza en los canales convencionales de comunicación los empleados deciden no informar a sus superiores sobre situaciones que son importantes para la empresa porque suponen que su voz no será escuchada.

\section{Grupo Experimental}

Con respecto al grupo experimental se nota un cambio positivo en cada una de las dimensiones. La percepción del clima laboral con respecto a cooperación y relaciones interpersonales aumentó en un 22\%. Este resultado muestra que fue acertada la manera de escoger las dimensiones del IMCOC a trabajar ya que se pudieron impactar de manera positiva y todas aumentaron después de aplicar la metodología. Este resultado es muy importante dentro de la investigación porque demuestra que si es posible mejorar el clima laboral, desde las variables de relacionamiento interpersonal y cooperación, al interior de la compañía y que la curva costo beneficio se adapta perfectamente ya que no hubo una inversión adicional ni en dinero ni en tiempo porque tanto el diseño como la aplicación de la metodología se hizo en horario laboral y en los tiempos establecidos de y para capacitación.

La figura 3 muestra claramente que el postest siempre fue superior al pretest.

1. Califique la ayuda y la colaboración que usted cree que existe entre

Figura 3.

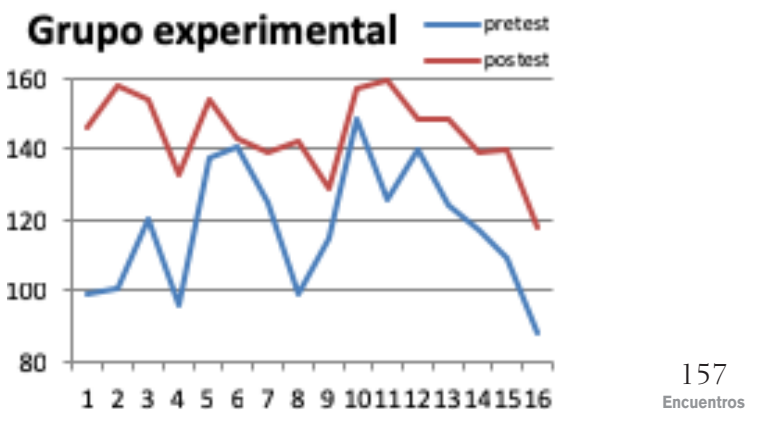


los trabajadores de esta empresa.

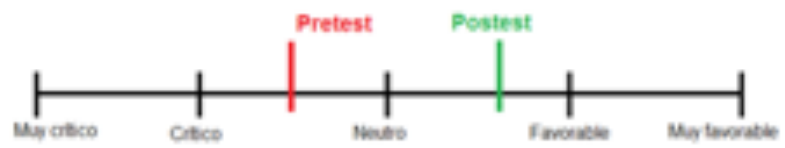

2. ¿En qué grado usted, ayuda y colabora con sus compañeros en el trabajo?

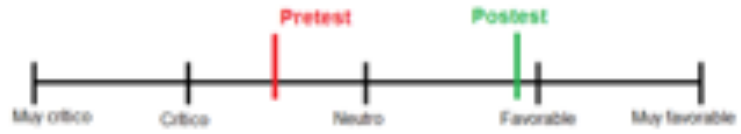

3. ¿Cómo califica su participación, si usted está vinculado en grupos de trabajo en la empresa?

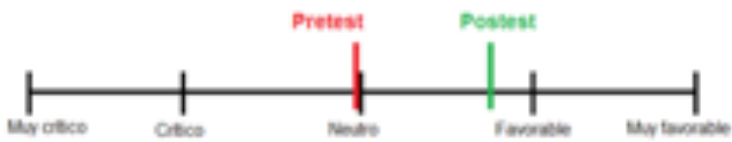

4. ¿Califique la frecuencia con que usted acostumbra divertirse con compañeros de su sección o de otra sección de la empresa?

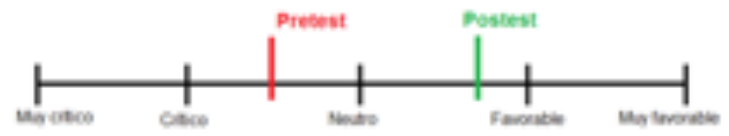

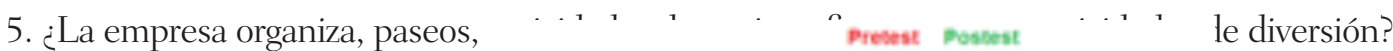

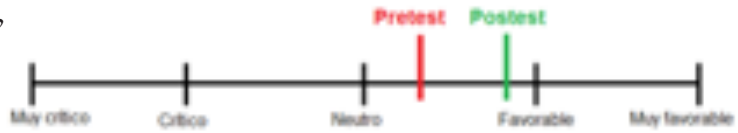

6. ¿Cómo califica usted, su part

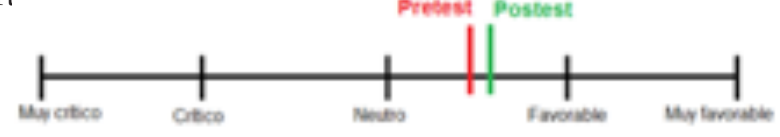

7. ¿Qué tanta ayuda le piden si

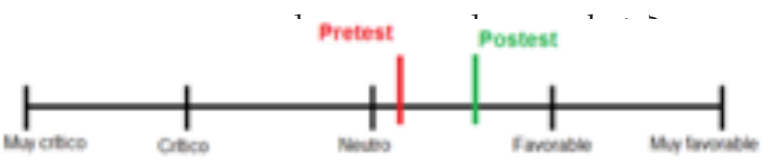

8. ¿Qué tanto participa usted, d

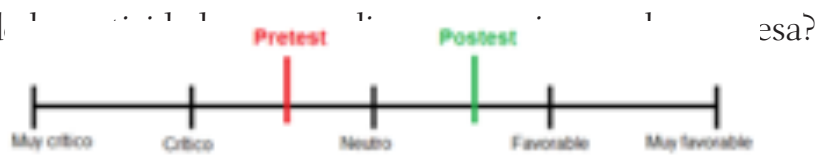


9. ¿Fuera de las horas de traba]

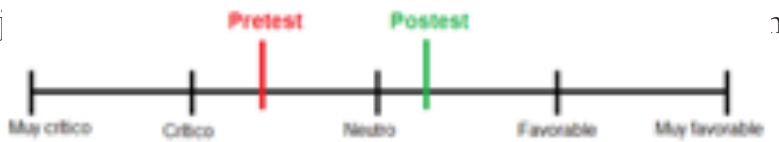

apañeros de labor?

10. ¿Califique la ayuda que ust $€$

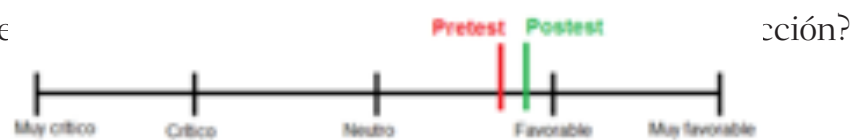

11. Cómo califica sus relacione

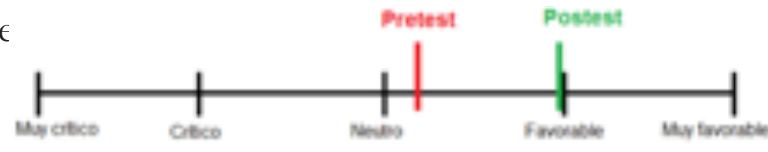

12. ¿Cuándo usted tiene un pr

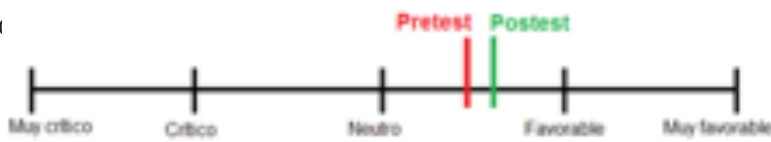

superiores?

13. ¿Cómo califica usted, el tra

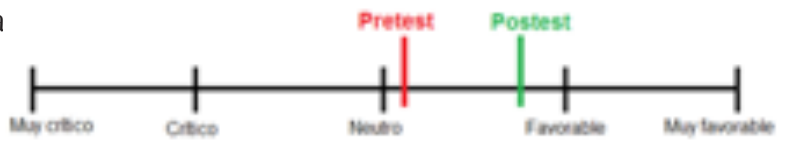

14. ¿Cómo califica usted, la co:

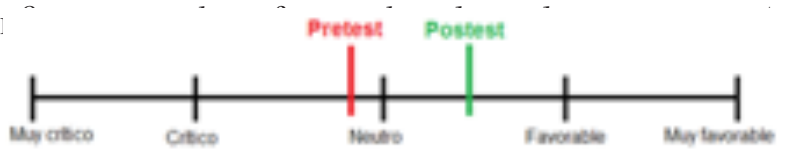

15. ¿Cuándo hay cambios en lá

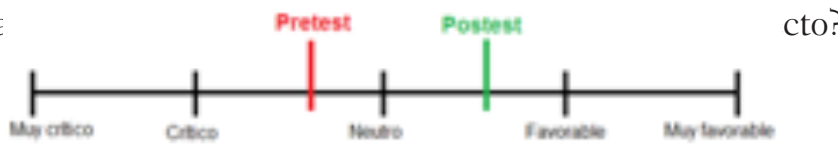

16. ¿En qué medida se enteran

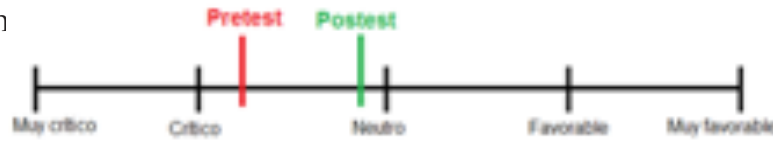




\section{Conclusiones}

Las conclusiones se han descrito teniendo en cuenta el alcance de los objetivos propuestos, tanto general como específico, para el proyecto de investigación:

- Se realizó la aplicación de actividades pedagógicas basadas en la pedagogía dialogante y técnicas de interacción grupal, de una estrategia de enseñanza - aprendizaje, desarrollada en siete talleres, encaminada a mejorar de manera directa el clima laboral de un grupo de 40 empleados de la empresa Claro Colombia con sede en la ciudad de Medellín. Esta estrategia constó de siete talleres organizados en un orden lógico que responden a la didáctica de aprendizaje de adultos en trabajo en equipo. La variación positiva en el grupo experimental fue del $22 \%$.

- Con los recursos que se contaba para la investigación, las dimensiones más apropiadas a impactar del IMCOC eran cooperación y relaciones interpersonales ya que no se requería de ningún factor externo al mismo relacionamiento de los trabajadores. Se pudo establecer con claridad que la esco- adecuada, así lo confirman las encuestas donde el postest del grupo experimental fue superior en cada uno de los reactivos al pretest.

- Pudo establecerse, según entrevistas realizadas durante la realización de los talleres, que en las relaciones laborales los individuos se desempeñan mejor cuando se sienten inmersos en ambientes interpersonales de aceptación y tolerancia. La confianza en sí mismos aumenta al sentirse parte activa del equipo de trabajo disminuyendo el miedo al rechazo por equivocaciones comunes y afianzando la comunicación organizacional, este último elemento con condiciones muy favorables para alcanzar los objetivos definidos por la empresa.

- La didácticas de enseñanza aprendizaje utilizadas al interior de la empresa, no con finalidad de aprendizaje sino de desarrollo humano, ofrecen un panorama mucho más extenso que solo la formación técnica tradicional. El desarrollo de las competencias socio afectivas favorece la adquisición de valores y conductas relacionados con el trabajo cooperativo. Esto se traduce al interior de la organización en la disminución de conflictos entre empleados y en una mayor productividad. Es por esta razón que se hace necesario dar un nuevo enfoque a las capacitaciones, entrenamientos e inducciones que se dictan actualmente en la empresa para incorporar los principios de una pedagogía dialogante que entienda que los conocimientos que debe adquirir el individuo para desempeñarse correctamente en su cargo deben basarse en las diversas dimensiones humanas.

\section{Referencias}

Álvarez, G. (2003). El clima organizacional en entidades educativas conceptualización investigaciones y resultados. Interamericana de Psicología ocupacional. Bogotá.

Amezola, J. J. H.; García, I. S. P., \& Castellanos, A. R. C. (2008). Desarrollo curricular por competencias profesionales integrales. Revista Educar. Recuperada del 6 de octubre del 2017. En línea: http://fcqi. tij.uabc.mx/documentos2010-2/VideoTutor\%20 Modelo\%20Educativo\%20UABC/Ramas/data/ downloads/formacion_por_competencias_ amezola_garcia.pdf

Argyris, C. (1957). Personality And Organization: The Conflict Between System And The Individual. Públicado en Administrative Science Quarterly. Vol. 1, No. 1 - Vol. 58, No. 4. Sage Publication. Cornell University.

Atkinson, J. W. (1964). An introduction to motivation. Van Nostrand. Atlanta.

Castañeda, C. (2014). Competencia Socio Afectiva en el Marco Escolar Colombiano. Escenarios. Vol. 12, No. 2, Julio-Diciembre de 2014, págs. 19-34. Recuperado del 14 de noviembre del 2014. En línea: file://C:/Users/Flex_Pentium/Downloads/312-6041-SM\%20(1).pdf

Cornell, F. G. (1955). Socially perceptive 
administration. The Phi Delta Kappan.

Chapman A. (2007). Maslow'sHierarchy of Needs. Documento en línea recuperado el 15 abril de 2011: www.businessballs.com/maslow.htm

Chiavenato, I. (2007). Administración de Recursos Humanos-el capital humano de las organizaciones. Mc Graw Hill. México D.F.

Colvin, M. \& Rutland, F. (2008). Is Maslow's Hierarchy of Needs a Valid Model of Motivation. LouisianaTechUniversity. Documento en línea recuperado 15 abril de 2011 de http://www.business. latech.edu/De Zubiría Samper, J. (2006). Los modelos pedagógicos: hacia una pedagogía dialogante. Editorial Magisterio. Bogotá.

Díaz Pinilla, M. (2010). 18 Factores de Evaluación de Clima Laboral. Gestión humana. Barranquilla.

Drucker, P. F. (1995). People and performance: The best of Peter Drucker on management. Routledge. Londres.

Duque, M. J. (2009). Desarrollo del modelo de clima organizacional Maquiavícola Recuperado el 5 de Marzo de 2012. Universidad Javeriana. En línea: http://www.javeriana.edu.co/biblos/tesis/ comunicacion/tesis307.pdf

Echeverría, R. (2003). Ontología del lenguaje. Editores JC Sáez. Santiago de Chile.

Edvinsson, L., \& Malone, M. S. (1998). El capital intelectual: cómo identificar y calcular el valor inexplorado de los recursos intangibles de su empresa. Grupo Editorial Norma. Bogotá

Fernández Collado, C., Hernández Sampieri, R., \& Baptista, P. (2006). Metodología de la Investigación. Mc Graw Hill. México.

García, N. T; Ochoa, L. E. (2004). Clima organizacional: promotor de la creatividad en la empresa. Revista Creando. Recuperado del 15 de abril del 2012. En línea: http://www.adriandejesusvargas. com/blog/el-clima-organizacional-como-promotorde-la-creatividad-en-la-empresa/

Gardner, H. (1998). Inteligencias múltiples. Editorial Paidós. Barcelona
Goleman, D. (2012). Inteligencia emocional. Editorial Kairós. Buenos Aires.

Gonczi, Andrew, (1996). Instrumentación de la educación basada en competencias. Perspectivas teóricas y prácticas en Australia. En Argüelles, A. (comp.), Competencia laboral y educación basada en normas de competencia, Limusa-sepcnccl-conalep, México, 1996.

Jaime, P. y Araujo, Y. (2009). Clima y cultura organizacional: ¿Dos constructos para explicar un mismo fenómeno? Decisiones basadas en el conocimiento y en el papel social de la empresa. XX Congreso anual de AEDEM, Vol. 1, 2007. Recuperado del 24 de julio del 2011. http://dialnet.unirioja.es/servlet/ articulo? codigo $=2486886$

Kaplún, M. (1997). De medios y fines en comunicación educativa. Chasqui. Revista Latinoamericana de Comunicación. Publicado en la Revista Chasqui. № 58. Recuperado del 6 de octubre del 2012. En línea: http://www.revistachasqui.org/ index.php/chasqui/article/view/1120

Kolb, D. A. (2014). Experiential learning: Experience as the source of learning and development. FT Press. New York.

Mansilla, D. (2007). Gestión organizacional elementos para su estudio. Editorial Universidad Católica de Chile. Santiago de Chile.

Maslow, A. H. (1991). Motivación y personalidad. Ediciones Díaz de Santos. Buenos Aires.

Maturana, H. (2002). El sentido de lo humano. Editorial Océano. Santiago de Chile.

Méndez, C. E. (2006). Clima Organizacional en Colombia: El IMCOC: Un método de análisis para su intervención. Editorial de la Universidad del Rosario. Bogotá.

Michael, Argyle. (1987). La psicología de la felicidad, Editorial Alianza. Madrid

Pérez, N. M. (2014). Caso aplicativo clima organizacional a la Dirección Nacional de Derecho de Autor bajo el método de medición IMCOC. Universidad
La Salle. Bogotá.

Ruiz, M., \& Moya, R. (1997). Los cuatro acuerdos. Amber Allen Publishing. California.

Santana, P. J., \& Cabrera, Y. A. (2007). Clima y cultura organizacional:; dos constructos para explicar un mismo fenómeno?. In Decisiones basadas en el conocimiento y en el papel social de la empresa. XX Congreso anual de AEDEM (p. 19). Asociación Española de Dirección y Economía de la Empresa (AEDEM).

Uribe, A. F. (2010). Estilos de liderazgo, riesgo psicosocial y clima organizacional en un grupo de empresas colombianas. Revista Facultad de Ciencias Económicas: Investigación y Reflexión. Universidad Militar de Nueva Granada. Bogotá. 